\title{
A CMB B-mode Search with Three Years of BICEP Observations
}

\author{
Colin Bischoff ${ }^{1}$ for the BICEP Collaboration \\ ${ }^{1}$ Harvard-Smithsonian Center for Astrophysics \\ 60 Garden St. MS 42, Cambridge, MA 02138, USA \\ email: cbischoff@cfa.harvard.edu
}

\begin{abstract}
The search for $B$-mode, or curl-type, polarization in the Cosmic Microwave Background is the most promising technique to constrain or detect primordial gravitational waves predicted by the theory of inflation. The BICEP telescope, which observed from the South Pole for three years from 2006 through 2008, is the first experiment specifically designed to target this signal. We review the observational motivations for inflation, the advantages of $B$-mode observations as a technique for detecting the gravitational wave background, and the design features of BICEP that optimize it for this search. The final analysis of all three seasons of BICEP data is in progress, representing a $50 \%$ increase in integration time compared to the result from Chiang et al. (2010). A preview of the three year result includes $E$-mode and $B$-mode maps, as well as the projected constraint on $r$, the tensor-to-scalar ratio.
\end{abstract}

Keywords. cosmology: observations, cosmic microwave background, polarization, instrumentation: polarimeters

\section{The Cosmic Microwave Background and Inflation}

The Cosmic Microwave Background (CMB) serves as a unique and invaluable window to our early universe. First discovered by Penzias and Wilson in 1964, the CMB is a relic thermal radiation that was originally emitted during the period of recombination, about 300,000 years after the Big Bang, when the universe had cooled enough that protons and electrons could form neutral Hydrogen. Recombination occurred at a temperature of $\sim 3,000 \mathrm{~K}$; since then, the size of the universe has increased by a factor of 1,100 , so the $\mathrm{CMB}$ is observed to have a temperature of $3 \mathrm{~K}$ today.

The temperature of the CMB is very nearly uniform across the entire sky, with small anisotropies at the $10^{-5}$ level. This uniformity poses a theoretical problem, because the causal horizon at the time of recombination corresponds to an angular scale of only three degrees. The question of how different regions of the universe that are causally disconnected achieved such similar temperatures is known as the horizon problem.

A solution to the horizon problem is offered by the theory of inflation. The theory proposes that the universe underwent a period of accelerated expansion for a tiny fraction of a second after the Big Bang (Guth, 1981). The expansion increased the scale factor of the space-time metric by 60 e-foldings, or $10^{26}$, transforming sub-atomic scales to astronomical ones. The observed temperature uniformity of the universe is explained because the regions that were causally disconnected at recombination were in close, causal proximity before inflation.

In addition to the horizon problem, there are several other observed features of our universe that are neatly explained by the theory of inflation.

- Observations of the CMB have demonstrated that the spatial geometry of the universe is flat on cosmological scales (de Bernardis et al., 2000). Inflation naturally explains 
this flatness, since our entire observable universe inflated from a small locally flat region of a pre-inflation space with arbitrary curvature.

- From temperature anisotropies in the CMB, we know that the initial density perturbations were nearly scale-invariant, with a small red tilt. Inflation naturally generates such a spectrum of perturbations, as quantum fluctuations are blown up to astronomical scales. After inflation ends, these initial perturbations will evolve linearly, starting from the time when their wavelength enters the causal horizon. The CMB encodes the state of each mode at the time of recombination.

- Related to the horizon problem is the presence of super-horizon modes in the CMB. The process described above to generate density perturbations will create modes with wavelength larger than the causal horizon. After inflation, these modes are frozen and unable to evolve, so we observe their primordial amplitudes.

- Guth's initial motivation to invent inflation did not involve the CMB at all; he proposed the theory to explain the observed lack of magnetic monopoles, which could have been diluted to unobservable density by the expansion of space.

While inflation does an impressive job of setting up initial conditions that match our universe, it remains a highly speculative theory. Proposed mechanisms for inflation all involve physics beyond the Standard Model. The energy scale involved, typically the Grand Unified Theory (GUT) scale $\left(10^{16} \mathrm{GeV}\right)$, is so enormous that direct experimental tests are impossible. However, an additional prediction of the theory of inflation is that it would create a cosmic background of gravitational waves, which would in turn leave an imprint on the Cosmic Microwave Background. Detecting the gravitational wave signal would provide a "smoking gun" for inflation, and is one of the most important goals in cosmology today.

\section{Searching for Inflation with CMB Polarization}

The amplitude of the gravitational wave background that is generated by inflation is parametrized by $r$, the tensor-to-scalar ratio, which specifies the power in gravitational waves (tensor perturbations) as a fraction of the power in density (scalar) perturbations (for details, see Baumann et al., 2009). The parameter $r$ depends on the energy scale of inflation, $V_{\phi}$, as shown in equation (2.1); inflation occurring at the GUT scale corresponds to a tensor-to-scalar ratio in the range 0.01 to 0.1 .

$$
V_{\phi} \simeq\left(3 \times 10^{16} \mathrm{GeV}\right) r^{1 / 4}
$$

The gravitational wave background does affect the power spectrum of the CMB temperature anisotropies. Experiments that map the CMB temperature have placed upper limits on $r$, with the best limit to date set at $r<0.17$ at $95 \%$ confidence by Keisler et al. (2011) using data from the South Pole Telescope in combination with full sky CMB maps from WMAP, measurements of baryon acoustic oscillations, and the Hubble parameter. This technique is limited, however, by the large amplitude, and correspondingly large sample variance, of the temperature power spectrum, which overwhelms the signal of interest.

The polarization of the CMB provides a way to search for the signal of inflation against a much lower background. An arbitrary polarization field can be decomposed into gradient-type and curl-type components (Kamionkowski, Kosowsky, and Stebbins, 1997, Hu and White, 1997), known respectively as $E$-modes and $B$-modes in analogy to electromagnetism. As illustrated in Figure 1, a plane-wave polarization fluctuation is a pure $E$-mode if the polarization is aligned parallel or perpendicular to the wave vector, $\vec{k}$; for a pure $B$-mode, the polarization is aligned at $\pm 45^{\circ}$ to the wave vector. 


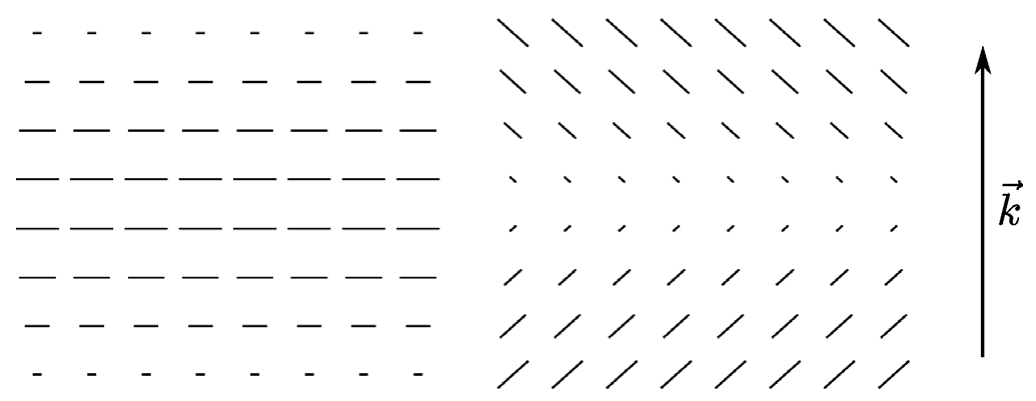

Figure 1. Pure $E$-mode (left) and $B$-mode (right) polarization patterns. For both cases, the wave vector of the modulation points vertically. $E$-mode polarization is parallel or perpendicular to $\vec{k} ; B$-mode polarization is oriented at $\pm 45^{\circ}$ to $\vec{k}$.

The density perturbations, which dominate the CMB temperature anisotropy, contribute only $E$-modes to the polarization anisotropy, while the gravitational wave background sources both $E$ and $B$-modes. This can be understood intuitively based on the fact that the odd-parity $B$-modes are characterized by a handedness in addition to their amplitude and wave vector; a scalar density perturbation has only amplitude and wave vector while tensor gravity waves also feature a polarization degree of freedom that can produce odd parity. With no contribution from density perturbations, a search for $B$ modes in the CMB polarization is a clean and powerful method to detect or limit the amplitude of gravitational waves sourced by inflation.

Figure 2 shows the current state of measurements of the $E$-mode and $B$-mode power spectra. The $E$-mode polarization was first detected by DAsI, observing from the South Pole, as reported in Kovac et al. (2002); this detection has since been confirmed by many other experiments. There have been no detections of $B$-mode polarization to date. The tightest upper limit on $B$-modes from gravitational waves comes from two seasons of BICEP data (Chiang et al., 2010), corresponding to $r<0.72$ at $95 \%$ confidence. This limit is weaker than the upper limit from CMB temperature measurements, but future polarization experiments will have the sensitivity to fully exploit the potential of $B$-modes in the search for inflation.

\section{The BICEP Telescope}

Background Imaging of Cosmic Extragalactic Polarization, or BICEP, is the first CMB telescope designed specifically to search for the $B$-mode signal of inflation. BICEP was initially deployed to the Amundsen-Scott South Pole Station in the austral summer of 2005-2006 and made observations during three winter seasons from 2006 through 2008. Results from the analysis of the first two observing seasons have been published in Chiang et al. (2010) along with a detailed instrument characterization in a companion paper, Takahashi et al. (2010). The $E$-mode power spectrum and $B$-mode upper limits from Chiang et al. are included in Figure 2.

A discussion of the design of the BICEP telescope highlights the challenges that must be addressed by any experiment targeting the $B$-mode signal.

- First, and most important, the signal is very small, so instrumental sensitivity is critical. The BICEP focal plane consists of 49 pairs of feedhorn-coupled polarization-sensitive bolometers (PSB), as shown in Figure 3. Orthogonal absorbers are each coupled to a neutron transmutation doped (NTD) Germanium bolometer and the two temperature 

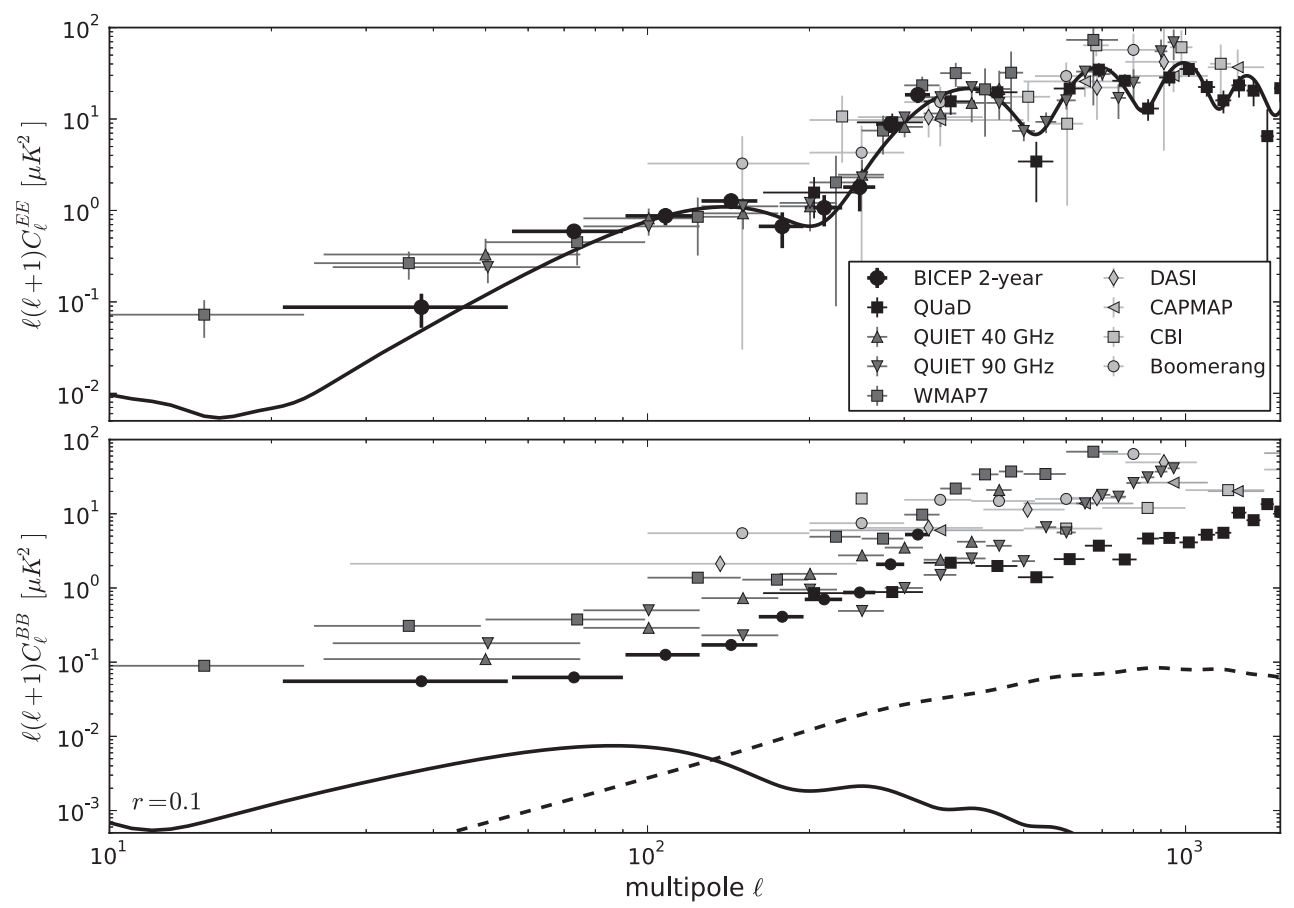

Figure 2. Measurements to date of the $E$-mode (top) and $B$-mode (bottom) power spectra, from Chiang et al. (2010), Brown et al. (2009), QUIET Collaboration (2011), QUIET Collaboration (2012), Larson et al. (2011), Leitch et al. (2005), Bischoff et al. (2008), Sievers et al. (2007), and Montroy et al. (2006). The $E$-mode spectrum has been detected at high signal-to-noise by many experiments, but only upper limits exist for the $B$-mode spectrum. The solid line shown for the $E$-mode spectrum is the theory curve for $\Lambda \mathrm{CDM}$ cosmology. The solid line shown for the $B$-mode spectrum is the expected signal from gravitational waves with $r=0.1$, peaking at $\ell \sim 80$, while the signal due to gravitational lensing of the $E$-modes, which peaks at $\ell \sim 10^{3}$, is shown as a dashed line.

measurements are differenced to obtain a measurement of linear polarization. The sensitivity of an individual PSB pair is background limited, so improved instrument sensitivity can only be achieved by increasing the number of detectors, or moving to a lower background environment, such as a stratospheric balloon or satellite.

- While $B$-modes are geometrically distinct, instrumental systematics can cause leakage of $E$-mode polarization or temperature anisotropies into the $B$-modes. The temperature power spectrum is five orders of magnitude larger than the $B$-mode spectrum corresponding to $r=0.1$, so these systematics must be very tightly controlled. The key to achieving this goal for BICEP lies in the clean design of the optical system - a cryogenic on-axis refractor with two plastic lenses cooled to $4 \mathrm{~K}$. A three axis mount allows BICEP to observe the same region of the sky at different boresight orientations; in combination with a highly redundant scan strategy, this provides additional suppression of systematics. The success of this optical design led to its adoption for successor projects, BICEP2 and the Keck Array.

- The $B$-mode signal from gravitational waves peaks at angular scales of $2-3^{\circ}$ (multipoles of 70-100), so requirements for angular resolution are modest. The aperture of the BICEP telescope is only $25 \mathrm{~cm}$, which yields beams with FWHM of $0.93^{\circ}$ and $0.60^{\circ}$ at 100 and $150 \mathrm{GHz}$, respectively. The small aperture means that the far-field of the optics is easily accessible for calibration. BICEP used a flat mirror to redirect the field of view 

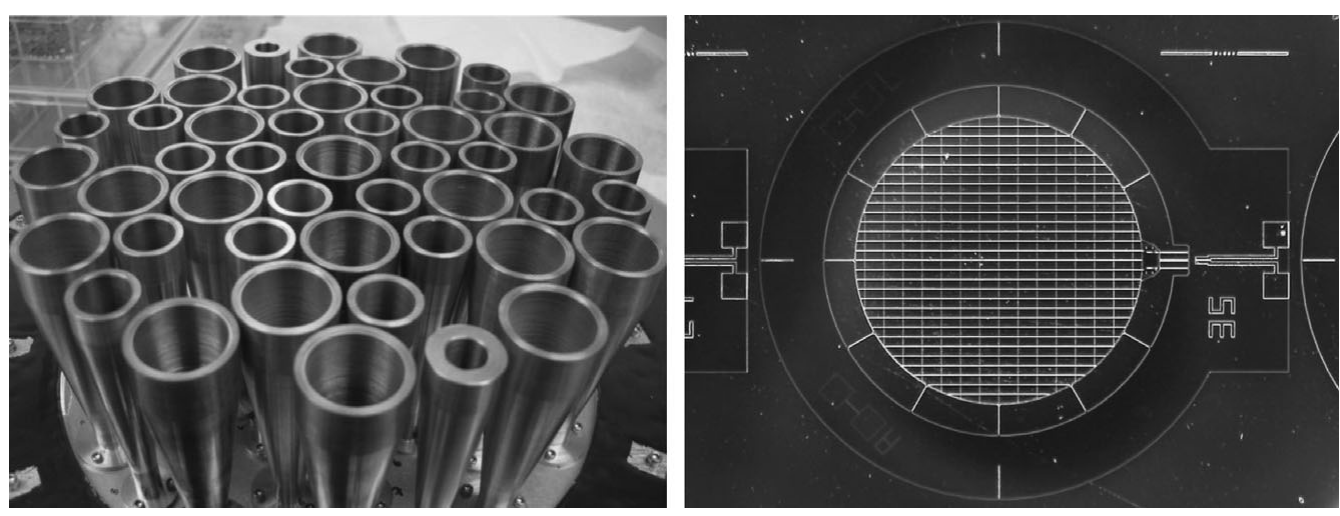

Figure 3. (left) The BICEP focal plane, consisting of 49 feedhorns - 25 operating at $100 \mathrm{GHz}$, 22 operating at $150 \mathrm{GHz}$, and 2 operating at $220 \mathrm{GHz}$. (right) A pair of polarization-sensitive bolometers, consisting of orthogonal absorbers (vertical and horizontal lines) each coupled to separate bolometers.

towards a calibration source mounted on an adjacent building. This technique yields high quality beam maps that are used to set upper limits on optical systematics.

- Galactic foreground signals, dominated by thermal dust at high frequencies and synchrotron emission at low frequencies, can be minimized by focusing observations on the cleanest regions of the sky. The South Pole observing site gives BicEP continuous access to the Southern Hole, two percent of the sky with uniquely low foregrounds. By observing at two frequencies, BICEP has a lever to distinguish between foreground and $\mathrm{CMB}$, if it proves necessary.

- Other advantages of observing from the South Pole include cold, dry atmospheric conditions, which provide the best microwave observing on Earth, and excellent logistics support from the United States Antarctic Program.

\section{Data Analysis for the BICEP Three-Year Result}

The result published in Chiang et al. used only the first two observing seasons (2006 and 2007); work is underway on a final analysis that will incorporate all three seasons of BICEP data. A change in the data selection will include several channels that were cut from the two-year result due to irregular bolometer time constants. Between the third season and the additional channels, the total integration time is increased by $50 \%$.

The new analysis will also incorporate a novel technique for removing temperature to polarization leakage due to instrumental systematics. This technique uses high signal-tonoise CMB temperature maps provided by WMAP (Jarosik et al., 2011) to deproject modes corresponding to certain classes of systematics. For example, a relative error in the calibrated responsivity between detectors in a PSB pair leads to direct coupling of the temperature map to polarization, known as "monopole leakage". A pointing offset between the two detectors in a pair results in false polarization signal proportional to the first spatial derivative of the temperature map, known as "dipole leakage". The deprojection procedure, described in detail in Aikin (2012, in preparation), applies a linear regression to determine the coefficient for each leakage mode over each observing phase (nine hours). The derived coefficients can be compared to other estimates of the instrumental systematics to make sure that the effect is well understood. While the deprojection procedure has been formulated for monopole, dipole, and quadrupole leakage (the last is due to differences in beam ellipticity between paired detectors), the instrument 

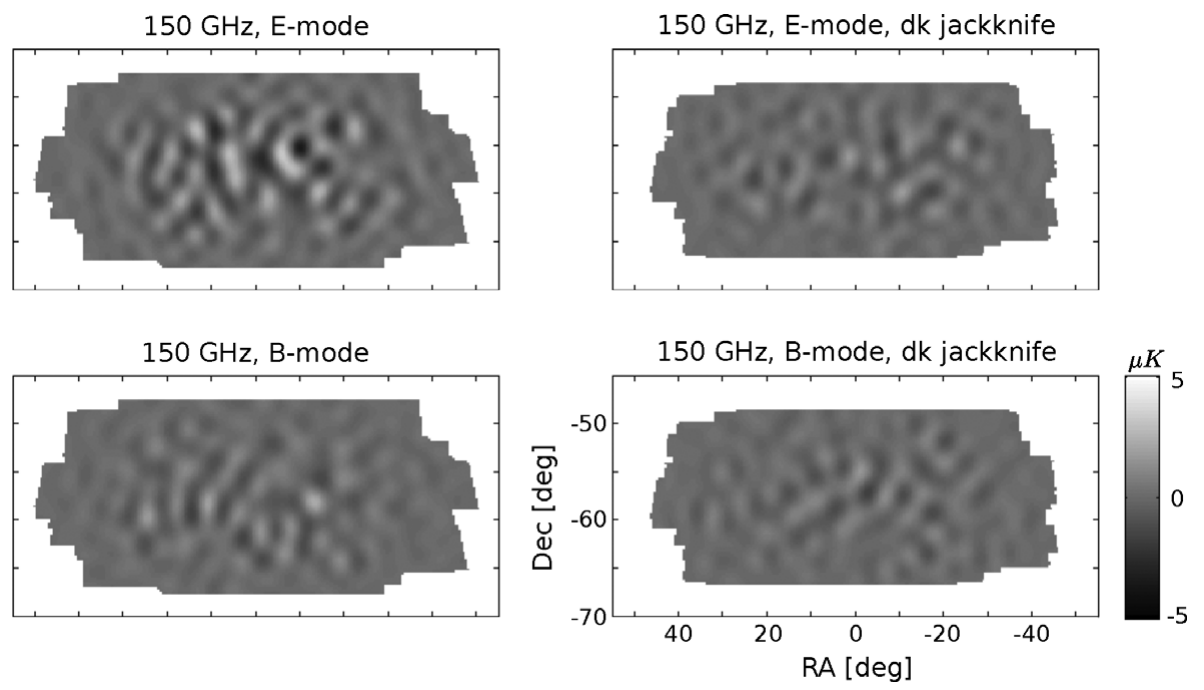

Figure 4. Maps of $E$-mode (top left) and $B$-mode (bottom left) polarization from three years of BICEP. Jackknife maps constructed by differencing data at alternate telescope boresight orientations are shown for both $E$-modes (top right) and $B$-modes (bottom right). All maps are consistent with noise, except for the non-jackknife $E$-modes.

characterization in Takahashi et al. (2010) demonstrated that only the monopole leakage exceeds (slightly) the benchmark corresponding to $r=0.1$. For this reason, the BICEP three-year analysis will deproject monopole leakage only. However, the well-characterized dataset has been invaluable for testing out the higher-order corrections that may prove necessary for more sensitive experiments.

Maps of $E$-mode and $B$-mode polarization made from three years of BICEP data are shown in Figure 4. An aggressive bandpass filter has been applied to show only modes in the multipole range of interest for the search for inflation, $50<\ell<120$. In addition to the actual $E$-mode and $B$-mode maps, two jackknife maps are shown, with $E$-modes and $B$ modes calculated by differencing maps made at alternate telescope boresight orientations. The sky signal subtracts out of the jackknife maps and residuals are found to be consistent with noise. Examination of Figure 4 confirms that the $E$-mode map shows significantly higher power, due to the real $\mathrm{CMB} E$-mode signal; the $B$-mode map is consistent with the jackknife maps, so no $B$-modes are detected.

While some work is still necessary before publication of the three-year CMB power spectra, simulations with realistic noise and correct data selection can be used to project the power of the result for constraining $r$. Figure 5 shows a histogram of $95 \%$ confidence upper limits derived from 500 independent simulations of the entire BICEP experiment. The solid grey bars include three years of data while the hollow bars include only the two years of data used for Chiang et al.. These histograms show that large variation in upper limits that can be obtained even for a non-detection, depending on the observed level of $B$-mode power. However, we do have the Chiang et al. result, indicated by the dashed vertical line, which is a strong prior on our expectation for the upper limit from the three-year analysis.

In conclusion, a final result for three years of BICEP will be published soon, with $50 \%$ additional integration time and improved systematics mitigation in comparison to Chiang et al.. However, this result will soon be eclipsed by vastly more sensitive data from BICEP2, which deployed during the austral summer of 2009-2010 and is currently in its third and final observing season, and the Keck Array, which deployed one year 


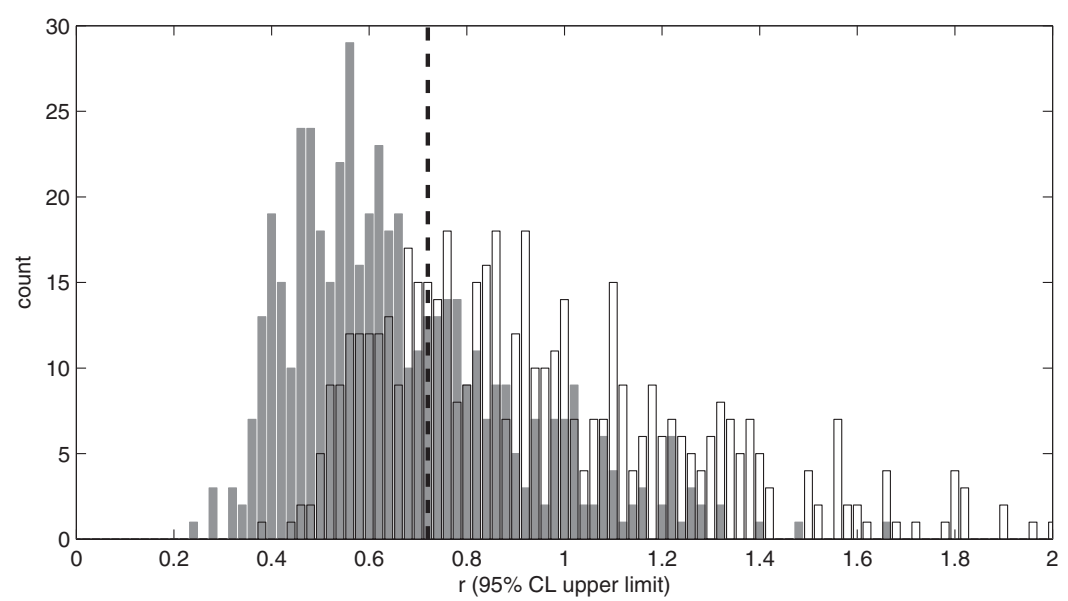

Figure 5. Histograms of $95 \%$ confidence upper limits on $r$, derived from simulations of the full three-year (solid grey bars) or two-year (hollow bars) BICEP data. The vertical dashed line indicates the upper limit actually obtained for the two year data, as published in Chiang et al. (2010).

after BICEP2 and will be operating through 2016. For more about BICEP2 and the Keck Array, see Clem Pryke's article in these proceedings.

\section{References}

Aikin, R., in preparation

Baumann, D. et al. 2009, AIP-CP, 1141, 10

Bischoff, C. et al. 2008, ApJ, 684, 771

Brown, M. L. et al. 2009, ApJ, 705, 978

Chiang, H. C. et al. 2010, ApJ, 711, 1123

de Bernardis, P. et al. 2000, Nature, 404, 955

Guth, Alan H. 1981, Phys. Rev. D, 23, 347

Hu, W. \& White, M. 1997, Phys. Rev. D, 56, 596

Jarosik, N. et al. 2011, ApJS, 192, 14

Kamionkowski, M., Kosowsky, A., \& Stebbins, A. 1997, Phys. Rev. D, 55, 7368

Keisler, R. et al. 2011, ApJ, 743, 28

Kovac, J. M. et al. 2002, Nature, 420, 772

Larson, D. et al. 2011, ApJS, 192, 16

Leitch, E. M. et al. 2005, ApJ, 624, 10

Montroy, T. E. et al. 2006, ApJ, 647, 813

Penzias, A. A. \& Wilson, R. W. 1964, ApJ, 142, 419

QUIET Collaboration 2011, ApJ, 741, 111

QUIET Collaboration 2012, arXiv:1207.5034

Sievers, J. L. et al. 2007, ApJ, 660, 976

Takahashi, Y. D. et al. 2010, ApJ, 711, 1141 